\title{
Milrinone for refractory cerebral vasospasm with delayed cerebral ischemia
}

\author{
Yasser B. Abulhasan, MBChB, FRCPC,, Johanna Ortiz Jimenez, MD, ${ }^{3}$ \\ Jeanne Teitelbaum, MD, FRCPC, ${ }^{1}$ Gabrielle Simoneau, PhD, ${ }^{4}$ and Mark R. Angle, MD ${ }^{1}$
}

${ }^{1}$ Neurological Intensive Care Unit and ${ }^{3}$ Department of Radiology, Montreal Neurological Institute and Hospital, McGill University, Montreal, Quebec, Canada; ${ }^{2}$ Faculty of Medicine, Health Sciences Center, Kuwait University, Kuwait; and ${ }^{4}$ Department of Epidemiology, Biostatistics and Occupational Health, McGill University, Montreal, Quebec, Canada

OBJECTIVE Intravenous (IV) milrinone is a promising option for the treatment of cerebral vasospasm with delayed cerebral ischemia $(\mathrm{DCl})$ after aneurysmal subarachnoid hemorrhage (aSAH). However, data remain limited on the efficacy of treating cases that are refractory to standard therapy with IV milrinone. The aim of this study was to determine predictors of refractory vasospasm/DCI despite treatment with IV milrinone, and to analyze the outcome of rescue therapy with intraarterial (IA) milrinone and/or mechanical angioplasty.

METHODS The authors conducted a retrospective cohort study of all patients with aSAH admitted between 2010 and 2016 to the Montreal Neurological Institute and Hospital. Patients were stratified into 3 groups: no DCl, standard therapy, and rescue therapy. The primary outcome was frequency of DCl-related cerebral infarction identified on neuroimaging before hospital discharge. Secondary outcomes included functional outcome reported as modified Rankin Scale (mRS) score, and segment reversal of refractory vasospasm.

RESULTS The cohort included 322 patients: 212 in the no DCI group, 89 in the standard therapy group, and 21 in the rescue therapy group. Approximately half $(52 \%, 168 / 322)$ were admitted with poor-grade aSAH at treatment decision (World Federation of Neurosurgical Societies grade III-V). Among patients with $\mathrm{DCl}$ and imaging assessing severity of vasospasm, $62 \%$ (68/109) had moderate/severe radiological vasospasm on DCl presentation. Nineteen percent $(21 / 110)$ of patients had refractory vasospasm/DCl and were treated with rescue therapy. Targeted rescue therapy with IA milrinone reversed $32 \%$ (29/91) of the refractory vasospastic vessels, and $76 \%$ (16/21) of those patients experienced significant improvement in their neurological status within 24 hours of initiating therapy. Moderate/severe radiological vasospasm independently predicted the need for rescue therapy (OR 27, 95\% $\mathrm{Cl} 8.01-112$ ). Of patients with neuroimaging before discharge, $40 \%$ (112/277) had developed new cerebral infarcts, and only $21 \%(23 / 112)$ of these were vasospasmrelated. Overall, $65 \%(204 / 314)$ of patients had a favorable functional outcome (mRS score $0-2$ ) assessed at a median of 4 months (interquartile range 2-8 months) after aSAH, and there was no difference in functional outcome between the 3 groups $(p=0.512)$.

CONCLUSIONS The aggressive use of milrinone was safe and effective based on this retrospective study cohort and is a promising therapy for the treatment of vasospasm/DCl after aSAH.

https://thejns.org/doi/abs/10.3171/2020.1.JNS193107

KEYWORDS milrinone; prognostic factors; subarachnoid hemorrhage; vasospasm; intracranial; vascular disorders

$\mathrm{A}$ NEURYSMAL subarachnoid hemorrhage (aSAH) is associated with high rates of morbidity and mortality. ${ }^{1,2}$ In addition to rebleeding, hydrocephalus, seizures, and healthcare-associated infections, symptom- atic cerebral vasospasm or clinical deterioration caused by delayed cerebral ischemia (DCI; vasospasm/DCI) is a common complication after aSAH, with limited effective therapeutic options. ${ }^{3-5}$ Based on a recent systematic re-

ABBREVIATIONS $\mathrm{ACA}=$ anterior cerebral artery; $\mathrm{aSAH}=$ aneurysmal $\mathrm{SAH} ; \mathrm{Cl}=$ confidence interval; $\mathrm{CTA}=\mathrm{CT}$ angiography; $\mathrm{DCl}=$ delayed cerebral ischemia; $\mathrm{DSA}=$ digital subtraction angiography; $E V D=$ external ventriculostomy drain; $I A=$ intraarterial; $I C A=$ internal carotid artery; ICU = intensive care unit; IQR = interquartile range; IV = intravenous; $\mathrm{LOS}=$ length of stay; MCA = middle cerebral artery; $\mathrm{mRS}=$ modified Rankin Scale; NICU = neurological ICU; OR = odds ratio; SAH = subarachnoid hemorrhage; $T C D=$ transcranial Doppler; WFNS $=$ World Federation of Neurosurgical Societies.

SUBMITTED November 24, 2019. ACCEPTED January 20, 2020.

INCLUDE WHEN CITING Published online March 27, 2020; DOI: 10.3171/2020.1.JNS193107. 
view, established predictors of DCI include large amounts of extravasated blood, poor clinical status on admission, smoking, hyperglycemia, hydrocephalus, diabetes mellitus, systemic inflammatory response syndrome, and a history of hypertension. ${ }^{6}$

Milrinone, a selective phosphodiesterase- 3 inhibitor associated with circulatory inotropic support and selective cerebral-vasodilatory effects, has recently emerged in a number of studies as a promising therapy for treating vasospasm/DCI. ${ }^{7-11}$ However, the modality of delivery and dosing differ among centers. ${ }^{12}$ Since 1998, our institution's protocol for the treatment of symptomatic cerebral vasospasm/DCI has been to administer an intravenous (IV) bolus followed by continuous IV perfusion of milrinone, with increases in dosage according to the patient's clinical response. The use of this protocol was associated with a lower incidence of both emergency angiograms and salvage angioplasty. ${ }^{13}$ In a recent retrospective French study comparing 1) the use of a combination of intraarterial (IA) milrinone infusion followed by IV milrinone until day 14 after aSAH, and 2) a continuous IV milrinone infusion for at least 7 days after aSAH, the authors reported similar efficacies between the two protocols. ${ }^{14}$ This study reported that $18 \%(15 / 77)$ of patients treated with IV milrinone needed rescue procedures. Although significant, there has been little information reported beyond this study regarding refractory DCI in patients treated with IV milrinone, and their ultimate outcomes following rescue procedures. ${ }^{14}$ To address this knowledge gap, we present our modern-day experience with IV milrinone as standard therapy and further address refractory cases that required endovascular treatment, specifically IA milrinone and/or angioplasty, as rescue therapy.

We report our 6-year experience of both standard therapy and rescue therapy use of milrinone for the treatment of vasospasm/DCI after aSAH, while focusing on patientrelated outcomes. We also explored predictors of vasospasm/DCI and refractoriness to standard therapy with IV milrinone treatment.

\section{Methods}

\section{Study Design and Patient Identification}

In this retrospective cohort study, we reviewed medical records from all patients admitted with spontaneous subarachnoid hemorrhage (SAH) between April 1, 2010, and March 31, 2016, to our neurological intensive care unit (NICU) at the Montreal Neurological Institute and Hospital in Montreal, Quebec, Canada. Patients were identified from the intensive care unit (ICU) databases and hospital registry. Inclusion criteria were all patients with aSAH proven by CT scan or CSF analysis, irrespective of timing between initial bleed and ICU admission. We excluded patients 1) admitted with nonaneurysmal, unspecified, or traumatic SAH, 2) who died within 3 days of SAH, or 3) who were treated with IA milrinone before receiving IV milrinone. Patients were then stratified by the presence or absence of DCI. Patients with DCI were further stratified into those who received standard therapy and those with refractory cases requiring rescue therapy.

\section{Patient Management}

Details of patient management have been previously published. ${ }^{13,15}$ In summary, all patients were admitted to the NICU. Patients received a standard protocol on admission, which included nimodipine (60 mg/4 hours) orally or via nasogastric tube, regular analgesia (acetaminophen \pm antiinflammatory medications), tranexamic acid to prevent ultra-early rebleed (maximum for 72 hours from ictus), antiepileptics (phenytoin) if required, and dexamethasone (2 mg twice daily) as third-line treatment for pain related to meningismus. Transcranial Doppler (TCD) ultrasonography and CT angiography (CTA) were performed upon clinical suspicion of vasospasm. IV and IA milrinone are part of our institution's therapy protocol for clinical deterioration caused by DCI in addition to maintaining euvolemia, normothermia, electrolyte homeostasis, and baseline blood pressure. The main modification to our previously published protocol ${ }^{13}$ was the option to escalate the dose of IV milrinone up to $2.5 \mathrm{mcg} / \mathrm{kg} / \mathrm{min}$.

For refractory cases, the rescue therapy protocol involved emergency angiography and IV or IA milrinone with or without angioplasty for one of the following reasons despite IV milrinone therapy: 1) new decline in neurological status, 2) persistent decline in neurological status, or 3) transiently symptomatic with worsening vasospasm on follow-up imaging.

\section{Collected Variables}

Collected variables included: demographics, admission characteristics, preexisting comorbidities, relevant laboratory tests, neurological status at treatment decision graded according to the World Federation of Neurosurgical Societies (WFNS) scale, morphological characteristics of SAH and incidence of vasospasm graded according to the modified Fisher scale, aneurysm morphology and treatment method, complications and supportive therapy throughout the ICU stay, ICU and hospital length of stay (LOS), last documented follow-up after discharge, and modified Rankin Scale (mRS) score within 18 months after aSAH. The mRS score was extrapolated from information on follow-up assessments by a neurologist (J.T.) certified in $\mathrm{mRS}$ scoring and blinded to patient grouping and hospitalization details. Favorable functional outcome was defined as an mRS score of $0-2$, and unfavorable functional outcome as an mRS score of 3-6.

\section{Vasospasm/DCl}

The detection of vasospasm/DCI was based on repeated neurological examination in the NICU and defined as new or deteriorating neurological signs unattributable to a cause other than DCI, with proven vasoconstriction by CTA, TCD, or digital subtraction angiography (DSA). A new decline in neurological status included changes in level of consciousness or orientation, a decrease in Glasgow Coma Scale score $\geq 2$, cranial nerve palsy, pronator drift, or focal motor deficit. ${ }^{5}$ We also included patients with new or deteriorating neurological signs unrelated to a cause other than DCI who immediately responded (i.e., within 1 hour) to the protocol therapy using milrinone and maintaining euvolemia, with or without vasopressors, but who 
did not have a CTA, TCD, or DSA performed to assess for vasoconstriction because symptoms and signs did not recur.

Neuroradiologists at our institution routinely classify vasospasm detected by DSA or CTA based on the degree of luminal narrowing of the vessel as none, mild $(<25 \%)$, moderate $(25 \%-50 \%)$, or severe $(>50 \%){ }^{16}$ For TCD, classification is based on the mean middle cerebral artery (MCA) velocity and MCA/internal carotid artery (ICA) mean velocity ratio, and stratified as either mild (> $120 \mathrm{~cm} / \mathrm{sec}$ and 3-4, respectively), moderate $(>120 \mathrm{~cm} /$ $\mathrm{sec}$ and 5-6, respectively) or severe $(>180 \mathrm{~cm} / \mathrm{sec}$ and $>$ 6 , respectively).${ }^{17}$ Severity of initial cerebral vasospasm was based on the reported assessment and retrospectively validated by the independent neuroradiologist blinded to patient grouping and hospitalization details.

\section{Neuroradiological Variables}

All available cranial CT and MRI scans after aSAH from before and after discharge were reviewed by the independent neuroradiologist (J.O.J., blinded to patient grouping and hospitalization details) to determine 1) the presence of existing and new cerebral infarcts at admission, 2) new cerebral infarcts before hospital discharge, 3) new cerebral infarcts after hospital discharge, and 4) external ventriculostomy drain (EVD) tract sequela. Infarcts were further stratified into vasospasm- and nonvasospasm-related. Vasospasm-related infarcts were defined as wedge-shaped cortical/subcortical hypodensities within the same vascular territory of the aneurysm, or infarcts located within the same vascular territory showing vasospasm on a previous CTA or DSA. ${ }^{18}$

For the rescue therapy cohort, angiograms from admission (baseline) and from before and after rescue therapy were evaluated by the independent neuroradiologist (unblinded to treatment) to obtain vessel caliber measurements. For the purpose of this analysis, significant vasospasm was defined as a 50\% decrease in vessel caliber compared with baseline measurements. ${ }^{19}$ Reversal of vasospastic arterial segments was defined as a 50\% increase in vessel caliber after rescue procedures compared with prerescue therapy measurements. ${ }^{14}$

\section{Outcomes}

Our primary outcome was cerebral infarction related to DCI, before hospital discharge. Secondary outcomes included mRS score within 18 months after SAH, cerebral infarction related to DCI after hospital discharge, reversal of refractory vasospastic segments following rescue therapy, in-hospital mortality, and ICU and hospital LOS.

\section{Statistical Analysis}

Analyses were conducted on data from the entire cohort of 322 patients. Collected variables were tabulated for the entire cohort and each patient group (no DCI, standard therapy, and rescue therapy). Patient characteristics and outcomes were summarized using median (interquartile range [IQR]) for continuous variables and frequency (percentage) for dichotomous or categorical variables. Adjustments for multiple testing were made when appropriate. ${ }^{20}$
Appropriate univariable tests were t-tests for continuous variables, chi-square tests for dichotomous or categorical variables, and Fisher exact tests if the chi-square test conditions were not met. Odds ratios (ORs) or regression coefficients, confidence intervals (CIs), and p values are reported.

Predictors of vasospasm/DCI and being refractory to IV milrinone were identified separately and compared to the no DCI group using appropriate univariable analyses. Multivariable analyses based on a logistic regression model were conducted for standard or rescue therapy separately, using strong predictors identified in the univariable analyses $(p<0.05)$. Associations between requiring standard or rescue therapy and the primary and secondary outcomes were investigated with logistic or linear regression models. Complete-case analyses were conducted.

The association between milrinone ceiling dose and severity of radiological vasospasm on DCI presentation categorized into mild, moderate, and severe was studied. The null hypothesis that the average ceiling dose of milrinone is equal across the three levels of severity of radiological vasospasm was tested using a 1-way ANOVA.

We also studied the causal relationship between milrinone ceiling dose and presence of cerebral infarct before hospital discharge and favorable outcome (mRS score 0-2) separately. Because patients were not randomized to receive low or high milrinone ceiling dose, propensity score weighting was performed. ${ }^{21}$ The propensity score represented the probability of receiving a high dose of milrinone $(>1.25 \mathrm{mcg} / \mathrm{kg} / \mathrm{min})$. The propensity score model was fitted with a logistic regression. The following predictors were selected based on expert opinion, and included in the propensity score model: age, WFNS grade, modified Fisher score, aneurysm treatment method, EVD, tranexamic acid, smoking status, history of hypertension, and history of cardiac disease. Once the propensity score was calculated, inverse probability of treatment weights was constructed for each patient. We used standardized mean difference to assess the balance of the predictors after applying the weights using a threshold of 0.10. Odds ratios were derived and robust standard errors were calculated. Further methodology, definitions, and statistical analysis details are found in the Supplemental Methods.

\section{Ethical Approval}

The McGill University Health Center Research Ethics Board approved this study. The board waived the need for patient consent because the data were derived from medical chart review. The procedures followed were in accordance with institutional guidelines.

\section{Results \\ Overall Population}

Over 6 years, we identified 445 patients admitted to the NICU with spontaneous SAH. Figure 1 describes the reasons for exclusion. We analyzed 322 patients with aSAH, of whom 110 (34\%) developed vasospasm/DCI (89 received standard therapy and 21 received rescue therapy). The median follow-up duration after discharge was 


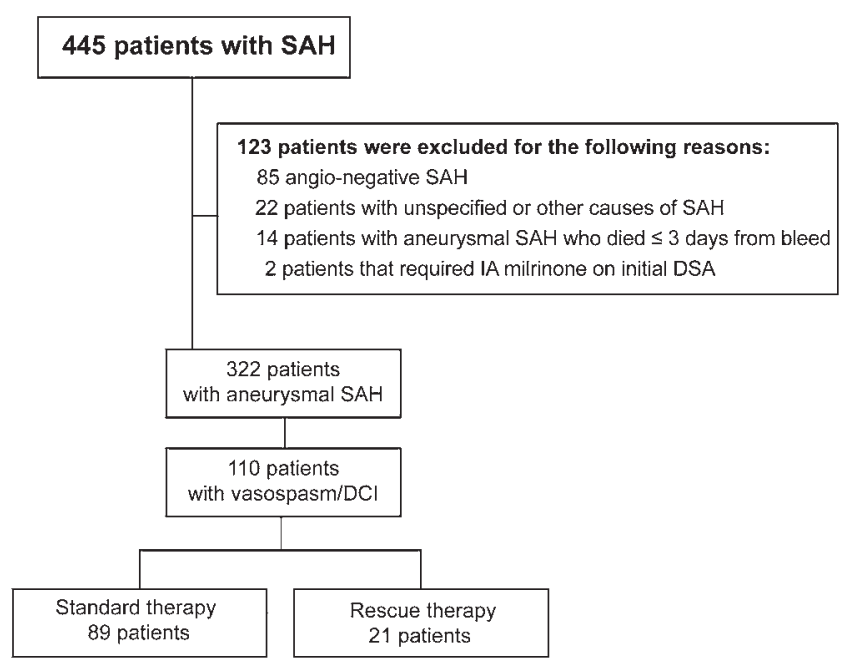

FIG. 1. Flowchart of patients in the study.

31 months (IQR 19-52 months). Missing data occurred sporadically, with the highest percentage missing for neuroimaging data.

Patient characteristics, stratified by no DCI, standard therapy, and rescue therapy groups, are presented in Table 1. Both median time from aSAH to admission and median time from admission to aneurysm treatment were 1 day (IQR 0-1 day). Fifty-two percent (168/322) of patients were admitted with poor-grade aSAH at treatment decision (WFNS grade III-V). Ten percent (33/322) were transferred to our institution more than 3 days after the initial bleed. IV milrinone was used to treat vasospasm/ DCI in 97\% (107/110) of cases for a median duration of 12 days (IQR 8-17 days), and vasopressors were used in $40 \%(44 / 110)$ of cases for a median of 4 days (IQR 2-9 days). Among patients with vasospasm/DCI, the detected vasospasm was mild in $36 \%$ (39/109), moderate in $27 \%$ (29/109), and severe in 36\% (39/109). Overall, 62\% (68/109) had moderate/severe radiological vasospasm on DCI presentation. Clinical DCI in the absence of neuroimaging was diagnosed in $3 \%(3 / 110)$ of patients. In the group without DCI, 27\% (57/212) of patients received IV milrinone for a median duration of 7 days (IQR 5-12 days) for various reasons; the primary reason was a presumptive diagnosis of DCI based on new onset of neurological symptoms and signs that was later proven to be due to other causes such as infection or metabolic derangement. Supplemental Table I shows milrinone use in all patients over the 6 years.

\section{Rescue Procedures for Refractory Vasospasm}

Of the 110 patients who had vasospasm/DCI, 21 (19\%) required a rescue endovascular procedure within 9 days of starting IV milrinone therapy (Table 2). Indications for rescue therapy despite IV milrinone therapy included: 1) new decline in neurological status $(81 \%, 17 / 21), 2)$ persistent decline in neurological status $(10 \%, 2 / 21)$, and 3) transiently symptomatic with worsening vasospasm on followup CTA during the ICU stay $(10 \%, 2 / 21)$. The median IV
TABLE 1. Patient characteristics

\begin{tabular}{|c|c|c|c|}
\hline \multirow[b]{2}{*}{ Variable } & \multirow[b]{2}{*}{$\begin{array}{l}\text { No DCl } \\
(n=212)\end{array}$} & \multicolumn{2}{|c|}{$\begin{array}{l}\text { Vasospasm/DCl } \\
\text { Therapy }\end{array}$} \\
\hline & & $\begin{array}{l}\text { Standard } \\
(\mathrm{n}=89)\end{array}$ & $\begin{array}{l}\text { Rescue } \\
(n=21)\end{array}$ \\
\hline \multicolumn{4}{|l|}{ Demographics } \\
\hline Median age (IQR), yrs & $57(49-69)$ & $54(48-62)$ & $52(49-56)$ \\
\hline Female, $\mathrm{n}(\%)$ & $144(68)$ & $57(64)$ & $15(71)$ \\
\hline Current smoker, n (\%) & $38(18)$ & $16(18)$ & $4(19)$ \\
\hline \multicolumn{4}{|l|}{$\begin{array}{l}\text { Preexisting comorbidities, } \\
\mathrm{n}(\%)\end{array}$} \\
\hline Hypertension & $83(39)$ & $36(40)$ & $8(38)$ \\
\hline Cardiac disease & $25(12)$ & $17(19)$ & $2(10)$ \\
\hline Diabetes mellitus & $15(7)$ & $6(7)$ & 0 \\
\hline \multicolumn{4}{|l|}{ Admission variable, $\mathrm{n}(\%)$} \\
\hline $\begin{array}{l}\text { WFNS grade at treatment } \\
\text { decision, III-V }\end{array}$ & $99(47)$ & $60(67)$ & $9(43)$ \\
\hline Modified Fisher score, 3-4 & 170/211 (81) & $83(93)$ & $17(81)$ \\
\hline Hydrocephalus & $92 / 211(44)$ & $36(40)$ & $11(52)$ \\
\hline $\begin{array}{l}\text { Presence of old cerebral } \\
\text { infarcts }\end{array}$ & $24 / 211(11)$ & $7(8)$ & 0 \\
\hline $\begin{array}{l}\text { Presence of new cerebral } \\
\text { infarcts }\end{array}$ & $5 / 211(2)$ & $3(3)$ & $3(14)$ \\
\hline Glucose $\geq 7 \mathrm{mmol} / \mathrm{L}$ & $109(51)$ & $55(62)$ & $8(38)$ \\
\hline $\begin{array}{l}\text { Sodium }<135 \mathrm{mmol} / \mathrm{L}, 0-3 \\
\text { days from aSAH }\end{array}$ & $46 / 211(22)$ & $17(19)$ & $7(33)$ \\
\hline \multicolumn{4}{|l|}{$\begin{array}{l}\text { Aneurysm location and mor- } \\
\text { phology, } \mathrm{n}(\%)\end{array}$} \\
\hline ACA rupture & $76(36)$ & $39(44)$ & $5(24)$ \\
\hline ICA rupture & $66(31)$ & $29(33)$ & $6(29)$ \\
\hline MCA rupture & $37(17)$ & $15(17)$ & $8(38)$ \\
\hline Posterior circulation rupture & $33(16)$ & $6(7)$ & $2(10)$ \\
\hline Maximal lumen size $\geq 7 \mathrm{~mm}$ & $86(41)$ & $35(39)$ & $5(24)$ \\
\hline Multiple aneurysms & $34(16)$ & $11(12)$ & $5(24)$ \\
\hline \multicolumn{4}{|l|}{$\begin{array}{l}\text { Aneurysm treatment method, } \\
\mathrm{n}(\%)\end{array}$} \\
\hline Coiling & $144(68)$ & $66(74)$ & $10(48)$ \\
\hline Clipping & $57(27)$ & $22(25)$ & $11(52)$ \\
\hline Unsecured & $11(5)$ & $1(1)$ & - \\
\hline \multicolumn{4}{|l|}{$\begin{array}{l}\text { Vasospasm/DCl character- } \\
\text { istics }\end{array}$} \\
\hline $\begin{array}{l}\text { Median onset from aSAH } \\
(\mathrm{IQR}) \text {, days }\end{array}$ & - & $6(4-8)$ & $5(5-8)$ \\
\hline $\begin{array}{l}\text { Mild radiological vaso- } \\
\text { spasm}^{*}\end{array}$ & $32 / 153(21)$ & $35 / 88(40)$ & $4(19)$ \\
\hline $\begin{array}{l}\text { Moderate radiological va- } \\
\text { sospasm}^{*}\end{array}$ & $15 / 153(10)$ & $24 / 88(27)$ & $5(24)$ \\
\hline $\begin{array}{l}\text { Severe radiological vaso- } \\
\text { spasm }^{*}\end{array}$ & $3 / 153(2)$ & $27 / 88(31)$ & $12(57)$ \\
\hline
\end{tabular}

CONTINUED ON PAGE $5 »$ 
» CONTINUED FROM PAGE 4

TABLE 1. Patient characteristics

\begin{tabular}{lrrr}
\hline & & \multicolumn{2}{c}{$\begin{array}{c}\text { Vasospasm/DCl } \\
\text { Therapy }\end{array}$} \\
\cline { 3 - 4 } \multicolumn{1}{c}{ Variable } & \begin{tabular}{c}
$\mathrm{No} \mathrm{DCl}$ \\
\cline { 3 - 4 }
\end{tabular} & $\begin{array}{c}\text { Standard } \\
(\mathrm{n}=212)\end{array}$ & $\begin{array}{c}\text { Rescue } \\
(\mathrm{n}=89)\end{array}$ \\
$(\mathrm{n}=21)$
\end{tabular}

* Two hundred sixty-two radiographic assessments on DCI presentation: 117 TCD ultrasonography, 107 CTA/perfusion, 38 DSA. Median duration from aSAH to imaging was 7 days (IQR $5-10$ days).

milrinone dose was $1.25 \mathrm{mcg} / \mathrm{kg} / \mathrm{min}$ (IQR $1-1.5 \mathrm{mcg} / \mathrm{kg} /$ $\mathrm{min}$ ) prior to rescue therapy and $1.75 \mathrm{mcg} / \mathrm{kg} /$ minute (IQR $1.5-2 \mathrm{mcg} / \mathrm{kg} / \mathrm{min}$ ) after rescue therapy.

In this cohort, there were 91 significant arterial vasospastic segments, with a median of 4 arterial segments (IQR 3-5 segments) per patient. The anatomical distribution of vasospastic segments by territory was MCA (n =37), anterior cerebral artery (ACA; $\mathrm{n}=36)$, ICA $(\mathrm{n}=$ $12)$, and posterior circulation $(n=6)$. These vasospastic segments required 31 targeted procedures, including 25 IA milrinone infusions (18 ICA, 4 MCA, 2 posterior circulation, and 1 common carotid artery) and 6 mechanical angioplasties (all MCA). Rescue procedures resulted in a significant increase in vessel caliber for 32\% (29/91) of the arterial segments, with 12 patients $(57 \%, 12 / 21)$ having at least 1 arterial segment reversed after therapy. Of the patients who did not have significant vasospastic segments reversed after therapy $(43 \%, 9 / 21), 56 \%$ (5/9) had neurological improvement within 24 hours (Table 2). Qualitative and objective assessments were performed and are shown in Supplemental Figure I. Overall, 76\% $(16 / 21)$ had significant improvement in their neurological status within 24 hours of initiating therapy. Only 1 patient had angiographically proven vasospasm recurrence in 4 arterial segments after rescue mechanical angioplasty (2 angioplasties were performed during the same session), requiring IA milrinone 6 days later. An additional 5 patients required mechanical angioplasty, 2 of whom underwent angioplasty followed by IA milrinone, and 2 required IA milrinone followed by angioplasty. One patient presented late and was found to have severe stenosis at the origin of the right vertebral artery, requiring angioplasty followed by stent-assisted coiling.

\section{Risk Factors for Refractory Vasospasm/DCI Requiring Rescue Therapy}

In patients with vasospasm/DCI, response to standard therapy was significantly associated with higher WFNS grade at treatment decision, higher modified Fisher score, and mild or moderate/severe vasospasm on radiological images, while we found a trend toward better response when the ruptured aneurysm was not located in the posterior circulation (Table 3). Higher WFNS grade at treatment decision as well as mild or moderate/severe vasospasm on radiological images remained significantly associated with requiring standard therapy in the multivariable analysis (Table 4). Moderate/severe vasospasm on radiological images was significantly associated with requiring rescue therapy, while there was a trend toward an association with younger age, presence of new cerebral infarct, the ruptured aneurysm being located in the MCA, and using clipping as the treatment method (Table 3). Only moderate/severe vasospasm on radiological images significantly predicted requiring rescue therapy in the multivariable analysis (Table 4).

\section{Milrinone Ceiling Dose}

Ninety-nine patients required standard or rescue therapy, had a record of milrinone ceiling dose, and had a record of severity of vasospasm at DCI presentation. The average dose of milrinone was $1.07 \pm 0.47 \mathrm{mcg} / \mathrm{kg} / \mathrm{min}$ in patients with mild radiological vasospasm, $1.08 \pm 0.40$ $\mathrm{mcg} / \mathrm{kg} / \mathrm{min}$ in patients with moderate severity, and $1.35 \pm$ $0.54 \mathrm{mcg} / \mathrm{kg} / \mathrm{min}$ in patients with severe radiological vasospasm. Average doses were significantly different across the three groups $(p=0.026)$. Results were similar when considering all patients who received milrinone $(n=107)$, although the association was only close to significant ( $\mathrm{p}$ $=0.05$ ).

A total of 145 patients had a record of milrinone ceiling dose and complete data on the two studied outcomes. After applying propensity score weighting, all predictors were balanced between patients receiving low versus high milrinone ceiling dose. In this sample, a higher dose of milrinone was associated with higher odds of developing cerebral infarct before hospital discharge (OR 1.66, 95\% CI 0.82-3.38) and lower odds of favorable outcomes (OR 0.76, 95\% CI 0.37-1.55), although neither association reached statistical significance $(\mathrm{p}=0.165$ and 0.454 , respectively).

\section{Complications}

Adverse effects of milrinone were uncommon despite IV doses of up to $2.5 \mathrm{mcg} / \mathrm{kg} / \mathrm{min}$. Complications in the 164 patients who received milrinone versus the 158 patients who did not receive milrinone (for whom data were available) included: 1) new or worsening arrhythmia in $7 \%(11 / 162)$ versus $9 \%(14 / 158) ; 2$ ) hypokalemia in $13 \%$ $(21 / 164)$ versus $20 \%(32 / 157)$; and 3$)$ pulmonary edema in $6 \%(10 / 164)$ versus 6\% (10/158), respectively. No patients required renal dialysis. 


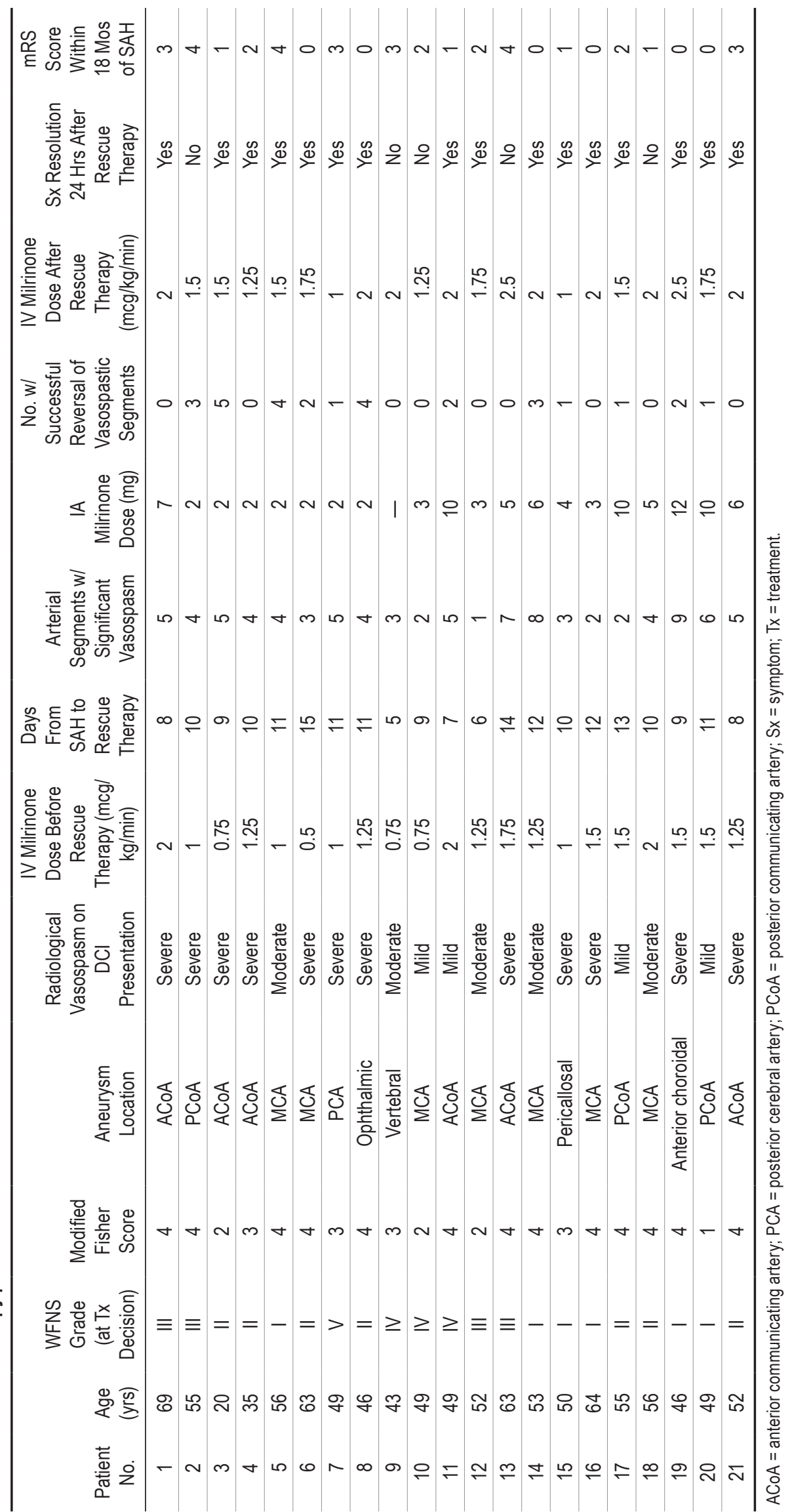




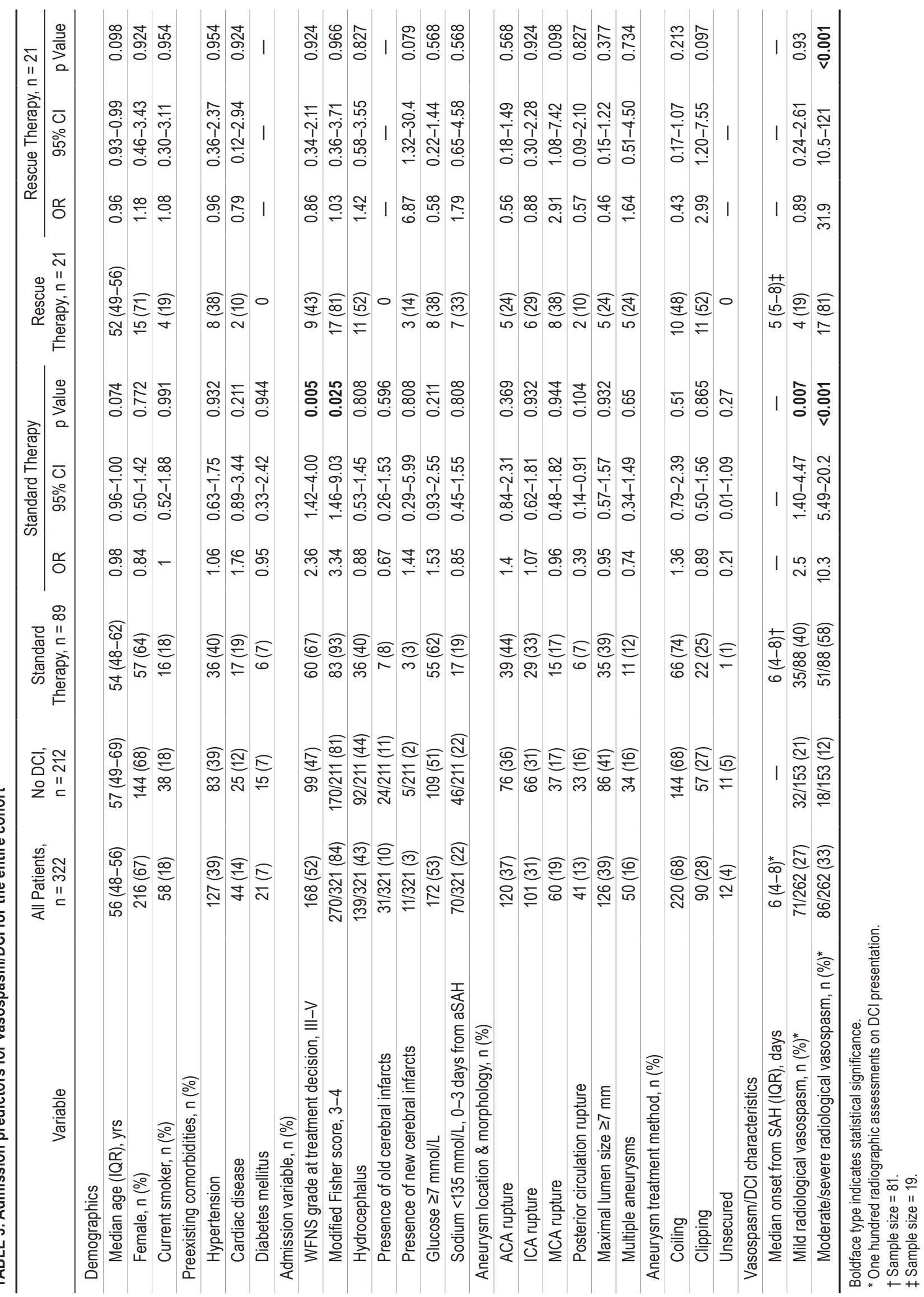


TABLE 4. Multivariable association of predictors with vasospasm/DCl for the entire cohort

\begin{tabular}{|c|c|c|c|c|c|c|}
\hline \multirow[b]{2}{*}{ Variable } & \multicolumn{3}{|c|}{ No DCl vs Standard Therapy $(n=240)^{*}$} & \multicolumn{3}{|c|}{ No $D C l$ vs Rescue Therapy $(n=173) \dagger$} \\
\hline & OR & $95 \% \mathrm{Cl}$ & $p$ Value & OR & $95 \% \mathrm{Cl}$ & p Value \\
\hline Age & 1.01 & $0.98-1.04$ & 0.435 & 0.99 & $0.95-1.04$ & 0.684 \\
\hline Higher WFNS grade at treatment decision & 2.32 & $1.10-4.97$ & 0.028 & & & \\
\hline Higher modified Fisher score & 2.11 & $0.68-6.98$ & 0.203 & & & \\
\hline PCA location & 0.73 & $0.19-2.80$ & 0.642 & & & \\
\hline Mild radiological vasospasm & 69.3 & $18.2-464$ & $<0.001$ & & & \\
\hline Moderate/severe radiological vasospasm & 142 & $36.8-961$ & $<0.001$ & 27 & $8.01-112$ & $<0.001$ \\
\hline New cerebral infarct & & & & 1.02 & $0.16-6.17$ & 0.987 \\
\hline MCA location & & & & 1.51 & $0.40-5.65$ & 0.54 \\
\hline Treatment method: clipping & & & & 1.01 & $0.27-3.58$ & 0.99 \\
\hline
\end{tabular}

Of the 164 patients who received milrinone, hypotension was noted in $15 \%(25 / 164)$ and severe hypotension in $2.4 \%$ (4/164). Two patients developed transient myocardial ischemia requiring either withholding milrinone therapy for 5 hours or decreasing the dose of milrinone infusion by half; 1 of the 2 patients had severe aortic stenosis on echocardiography. In the rescue therapy group, none of the rescue procedures resulted in iatrogenic complications. The associations of complications and supportive therapies with requiring standard or rescue therapy are shown in Table 5.

\section{Outcomes}

Of the 277 patients with neuroimaging before discharge, $40 \%(112 / 277)$ developed new cerebral infarcts, but only $21 \%$ (23/112) of these were vasospasm-related (Table 6). Of the infarcts unrelated to vasospasm, 43 were posthemor- rhage reabsorption, 40 were procedure-related (22 clipping and 18 coiling), 1 was a thrombotic complication, and 5 had no clear underlying cause. Postdischarge, 11 of 187 patients with available neuroimaging developed new infarcts, of which $2(1 \%)$ were vasospasm-related and were detected 3 and 10 months from aSAH (due to unavailability of the prior image). Of the 9 nonvasospasm-related infarcts, 4 were procedure-related (detected after 3 months either when the prior image was unavailable or inadequate technique that did not allow infarcts to be detected earlier), 2 were secondary to hemorrhage reabsorption, and 3 had no cause determined. Postdischarge neuroimaging was acquired a median of 15 months after aSAH (IQR 9-20 months).

Overall, 65\% (204/314) of patients had a favorable functional outcome (mRS score 0-2) assessed at a median of 4 months after aSAH (IQR 2-8 months). There was no difference in functional outcome among the 3 groups ( $\mathrm{p}$ $=0.512$, Fisher's exact test). On average, patients requir-

TABLE 5. Univariable association of complications and supportive therapies with vasospasm/DCI for the entire cohort

\begin{tabular}{|c|c|c|c|c|c|c|c|c|c|c|}
\hline \multirow[b]{2}{*}{ Variable } & \multirow{2}{*}{$\begin{array}{c}\text { All Patients, } \\
n=322\end{array}$} & \multirow{2}{*}{$\begin{array}{l}\text { No } D C l \\
n=212\end{array}$} & \multirow{2}{*}{$\begin{array}{c}\text { Standard } \\
\text { Therapy, } n=89\end{array}$} & \multicolumn{3}{|c|}{ Standard Therapy } & \multirow{2}{*}{$\begin{array}{c}\text { Rescue } \\
\text { Therapy, } n=21\end{array}$} & \multicolumn{3}{|c|}{ Rescue Therapy } \\
\hline & & & & OR & $95 \% \mathrm{Cl}$ & p Value & & OR & $95 \% \mathrm{Cl}$ & $p$ Value \\
\hline Rebleed & 34 (11) & $23(11)$ & $8(9)$ & 0.81 & $0.33-1.82$ & 0.808 & $3(14)$ & 1.37 & $0.30-4.45$ & 0.924 \\
\hline Tranexamic acid & $223(69)$ & $141(67)$ & $64(72)$ & 1.29 & $0.76-2.25$ & 0.596 & $18(86)$ & 3.02 & $0.98-13.2$ & 0.245 \\
\hline Intubation & $162(50)$ & $97(46)$ & $54(61)$ & 1.83 & $1.11-3.05$ & 0.052 & $11(52)$ & 1.3 & $0.53-3.26$ & 0.924 \\
\hline EVD & $191(59)$ & $114(54)$ & $65(73)$ & 2.33 & $1.38-4.05$ & 0.007 & $12(57)$ & 1.15 & $0.47-2.92$ & 0.924 \\
\hline Mannitol & $64(20)$ & $31(15)$ & $29(33)$ & 2.82 & $1.57-5.08$ & 0.003 & $4(19)$ & 1.37 & $0.38-4.01$ & 0.924 \\
\hline $3 \%$ saline & $166(52)$ & $83(39)$ & $68(76)$ & 5.03 & $2.91-8.99$ & $<0.001$ & $15(71)$ & 3.89 & $1.51-11.3$ & 0.074 \\
\hline Sodium, <130 mmol/L & 46/321 (14) & $19 / 211(9)$ & $21(24)$ & 3.12 & $1.58-6.20$ & 0.005 & $6(29)$ & 4.04 & $1.32-11.3$ & 0.077 \\
\hline Potassium, $<3.0 \mathrm{mmol} / \mathrm{L}$ & $53 / 321(17)$ & $35 / 211(17)$ & $14(16)$ & 0.94 & $0.46-1.81$ & 0.932 & $4(19)$ & 1.18 & $0.33-3.43$ & 0.924 \\
\hline Phenytoin & $164(51)$ & $95(45)$ & $59(66)$ & 2.42 & $1.46-4.10$ & 0.004 & $10(48)$ & 1.12 & $0.45-2.77$ & 0.924 \\
\hline Milrinone & $164(51)$ & $57(27)$ & $86(97)$ & 78 & $27.8-326$ & $<0.001$ & $21(100)$ & - & - & \\
\hline Vasopressors & $81(25)$ & $37(17)$ & $34(38)$ & 2.92 & $1.68-5.11$ & 0.001 & $10(48)$ & 4.3 & $1.68-10.9$ & 0.033 \\
\hline Steroids & $133(41)$ & $68(32)$ & $53(60)$ & 3.12 & $1.88-5.24$ & $<0.001$ & $12(57)$ & 2.82 & $1.14-7.23$ & 0.098 \\
\hline
\end{tabular}

Data presented as number (\%) for the complete data set unless otherwise specified.

Boldface type indicates statistical significance. 


\begin{tabular}{|c|c|c|c|c|c|c|c|c|c|c|}
\hline \multirow[b]{2}{*}{ Variable } & \multirow[b]{2}{*}{ All Patients } & \multirow[b]{2}{*}{ No DCl } & \multicolumn{4}{|c|}{ Standard Therapy } & \multicolumn{4}{|c|}{ Rescue Therapy } \\
\hline & & & $\begin{array}{l}\text { Standard } \\
\text { Therapy }\end{array}$ & OR & $95 \% \mathrm{Cl}$ & $p$ Value & $\begin{array}{l}\text { Rescue } \\
\text { Therapy }\end{array}$ & OR & $95 \% \mathrm{Cl}$ & p Value \\
\hline \multicolumn{11}{|l|}{ Neurology data, n (\%) } \\
\hline $\begin{array}{l}\text { Vasospasm-related cerebral } \\
\text { infarct before hospital } \\
\text { discharge }\end{array}$ & $23 / 277(8)$ & $4 / 173(2)$ & $13 / 85(15)$ & 7.62 & $2.41-24.2$ & $<0.001$ & $6 / 19(32)$ & 19.5 & $4.88-77.9$ & $<0.001$ \\
\hline $\begin{array}{l}\text { Nonvasospasm-related } \\
\text { cerebral infarcts before } \\
\text { hospital discharge }\end{array}$ & $89 / 277(32)$ & $60 / 173(35)$ & $25 / 85(29)$ & 0.78 & $0.45-1.38$ & 0.398 & $4 / 19(21)$ & 0.5 & $0.16-1.58$ & 0.239 \\
\hline $\begin{array}{l}\text { EVD tract hemorrhage } \\
\text { sequala }\end{array}$ & $102 / 183(56)$ & $56 / 108(52)$ & $40 / 64(63)$ & 1.55 & $0.82-2.91$ & 0.175 & $6 / 11(55)$ & 1.11 & $0.32-3.87$ & 0.865 \\
\hline $\begin{array}{l}\text { Vasospasm-related cerebral } \\
\text { infarct 3-12 mos after aSAH }\end{array}$ & $2 / 187$ (1) & 0 & $1 / 59(2)$ & - & - & - & $1 / 16(6)$ & - & - & - \\
\hline $\begin{array}{l}\text { Nonvasospasm-related } \\
\text { cerebral infarcts 3-12 mos } \\
\text { after SAH }\end{array}$ & $9 / 187(5)$ & $6 / 112(5)$ & $2 / 59(3)$ & 0.62 & $0.12-3.17$ & 0.566 & $1 / 16(6)$ & 1.18 & $0.13-10.5$ & 0.883 \\
\hline \multicolumn{11}{|l|}{ Outcomes } \\
\hline $\begin{array}{l}\text { Favorable outcome, mRS } \\
\text { score } 0-2, n(\%)\end{array}$ & $204 / 314(65)$ & $129 / 206(63)$ & $61 / 87(70)$ & 1.4 & $0.82-2.40$ & 0.221 & $14(67)$ & 1.19 & $0.46-3.09$ & 0.715 \\
\hline In-hospital mortality, n (\%) & $35(11)$ & $27(13)$ & $8(9)$ & 0.68 & $0.29-1.55$ & 0.357 & 0 & - & - & - \\
\hline $\begin{array}{l}\text { Median ICU LOS (IQR), } \\
\text { days }\end{array}$ & $14(6-21)$ & $9(4-16)$ & $19(14-24)$ & $8.08^{*}$ & $6.04-10.1$ & $<0.001$ & $22(20-25)$ & $11.5^{*}$ & $7.82-15.2$ & $<0.001$ \\
\hline $\begin{array}{l}\text { Median hospital LOS (IQR), } \\
\text { days }\end{array}$ & $22(11-33)$ & $16(8-27)$ & $26(20-35)$ & $4.64^{*}$ & -5.87 to 15.1 & 0.386 & $33(25-40)$ & $20.1^{*}$ & $1.05-39.1$ & 0.039 \\
\hline
\end{tabular}

Boldface type indicates statistical significance.

${ }^{*}$ Regression coefficients.

ing standard and rescue therapy stayed, respectively, 8 days (95\% CI 6-10 days) and 12 days (95\% CI 8-15 days) longer in the ICU than patients with no vasospasm, and also had a significantly longer hospital stay. Further results including missing data, neuroradiological data, complications, supportive therapy, and causes of in-hospital mortality are found in the Supplemental Results.

\section{Discussion}

We conducted a large single-center retrospective cohort study to assess the efficacy and outcome of treating refractory vasospasm/DCI cases in a homogeneous population of patients with aSAH over a 6-year period. The main findings of our study were: 1) despite $52 \%$ of patients being admitted with poor-grade aSAH, only 8\% (23/277) developed vasospasm-related cerebral infarcts before hospital discharge, and 65\% (204/314) of patients had a favorable functional outcome; 2) moderate/severe vasospasm was significantly associated with the need for rescue therapy; and 3) aggressive use of milrinone is safe.

In our cohort, 34\% of patients developed vasospasm/ DCI, and of these 19\% did not respond to standard therapy with IV milrinone. Of those who were refractory to standard therapy, $76 \%$ had significant improvement in their neurological status within 24 hours of initiating treatment with IA milrinone therapy with or without angioplasty, and the functional outcome was favorable in $67 \%$ of patients.
The outcome in our refractory DCI group treated with rescue therapy was much better than the outcome found in a study by Suwatcharangkoon et al., ${ }^{22}$ in which DCI was initially treated with a hypertensive agent alone (no milrinone therapy), and refractory DCI was treated with angioplasty. The good outcome in their refractory group was defined as an mRS score of $0-3$ at 12 months compared to our definition of $0-2$ at 18 months, yet their proportion of patients with good outcome was $38 \%$ compared to our $67 \%$. This suggests that milrinone is likely beneficial in both responsive and refractory DCI, when administered intravenously as well as intraarterially, although improvement over time (from 12 to 18 months after the event) may play a role as well.

Among our patients with refractory vasospasm/DCI who underwent neuroimaging before discharge, only $32 \%$ developed DCI-associated cerebral infarcts, and the proportion of patients with favorable functional outcome by mRS score was comparable between the aSAH groups with and without DCI. In previous studies, up to $45 \%$ of patients with DCI (including refractory and responsive cases) developed delayed strokes that were responsible for severe disabilities. ${ }^{23-25}$ Not only was the number of secondary strokes higher in these patients than in our cohort, their outcome was also worse. Vergouwen et al. in $2011^{26}$ found that of 194 patients with moderate to severe vasospasm, $43 \%$ had neurological worsening of any cause, $20 \%$ had cerebral infarction, and cerebral infarction contributed 

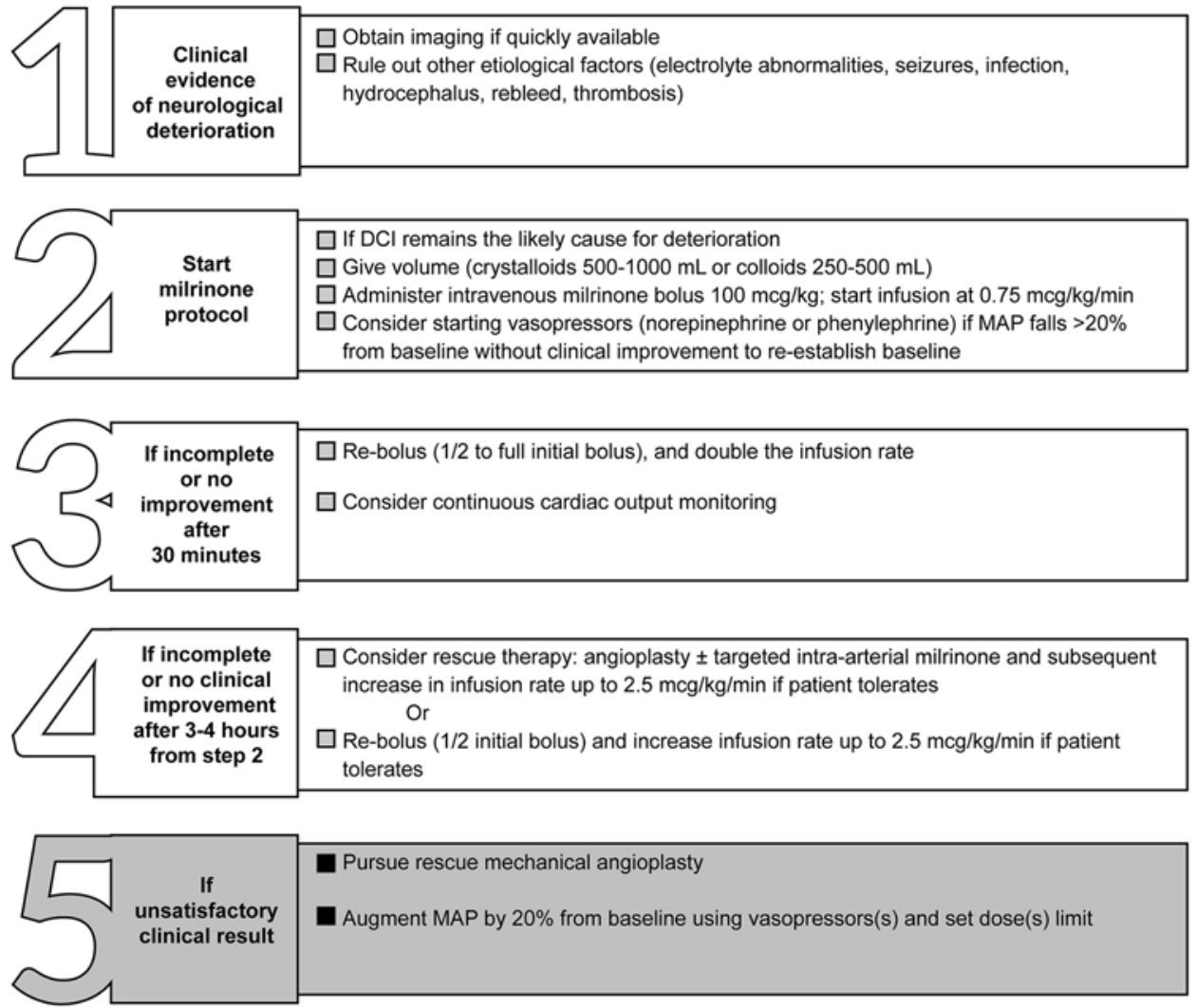

FIG. 2. Milrinone protocol. MAP = mean arterial pressure.

to unfavorable outcome by vasospasm-dependent and -independent effects. Milrinone may be the reason for the decrease in delayed strokes and improved outcome observed in our study, because it acts not only as a vasodilator but also as an antiinflammatory agent through the inhibition of phosphodiesterase- $3 .^{13}$ The improvement in neurological status in refractory cases was mainly seen within 24 hours of initiating therapy, even though the change in vessel caliber can be seen within minutes (data not shown). This finding is another indication that there may be more than one mechanism of action for milrinone, or that the increase in cerebral perfusion after dilation requires time to reverse the effects of the transient ischemia.

We found that younger age, higher WFNS grade at treatment decision, higher modified Fisher score, location of ruptured aneurysm, and mild and moderate/severe vasospasm on radiological images were all significantly associated with vasospasm/DCI responding to standard therapy. These findings are consistent with those of recent studies, ${ }^{27,28}$ including a study by Lee et al., ${ }^{29}$ but we did not find associations with smoking, hyperglycemia, hydrocephalus, diabetes mellitus, or a history of hypertension as noted by de Rooij et al. in their systematic review. ${ }^{6}$ The only independent predictor of refractory vasospasm/DCI that we identified in our study was moderate/severe radiological vasospasm on neuroimaging at the time of DCI presentation. This finding was not unexpected, nor was it surprising that the odds of developing new vasospasmrelated cerebral infarcts also increased with the severity of the vasospasm/DCI, which fortunately did not translate into worse functional outcome, as mentioned previously.

In our institution's protocol, we use milrinone quite aggressively to treat DCI (Fig. 2). Our IV dose is much higher than that normally used in cardiology, and higher than the dose used in most centers using milrinone for DCI. ${ }^{30}$ The IA dose (2-12 mg total dose) is commensurate with that used by others, but is always used in our institution in conjunction with a high starting dose of IV milrinone that can be further increased if necessary. In our previous study, the side effects of this regimen were negligible..$^{13}$ In our present study, although high doses of IV milrinone were used, serious complications were uncommon. Moreover, the propensity score analysis found no evidence of a causal relationship between high ceiling doses of IV milrinone and worse outcomes. Therefore, there is little reason to avoid using milrinone in patients with DCI, despite the lack of a randomized controlled trial.

This study has several limitations. First, the presented data are retrospective and derived from a single specialized tertiary care center, limiting generalizability. Second, the mRS score used to assess functional outcome was retrospectively calculated from medical records, making it less accurate than directly interviewing the patient. However, the single neurologist who extracted the information was blinded to hospitalization details and patient grouping. Third, it is difficult to know which complications are due to milrinone because SAH itself is a cause of myocardial stunning, signs of myocardial ischemia, pulmonary 
edema, and arrhythmia. Fourth, the found associations between requiring standard or rescue therapy are not interpreted as causal relationships but rather describe clinical practice and treatment decisions. Therefore, confounding is not an issue. However, the propensity score analysis of milrinone ceiling dose and two outcomes relied on the assumption of no unmeasured confounders. Given the large amount of data collected on patients, we are confident that this assumption is reasonable. Finally, neuroimaging at discharge was only available in $86 \%$ (277/322) of the patients included in the analysis.

\section{Conclusions}

The aggressive use of milrinone for the treatment of refractory vasospasm/DCI after aSAH remains a promising and effective therapy option. Despite the modest reversal rate of significant vasospasm segments during targeted IA rescue therapy, neurological improvement was evident within 24 hours of initiating therapy. Additionally, the patient-related outcomes in our cohort were very good overall despite the high percentage of patients who presented with poor WFNS grade at treatment decision. A randomized controlled trial would best validate our findings.

\section{Acknowledgments}

The authors acknowledge the work of Andréane Massicotte, Anne-Sophie Châtillon-Angle, and Cedric Châtillon-Angle for their invaluable role in data extraction.

\section{References}

1. Go AS, Mozaffarian D, Roger VL, et al. Heart disease and stroke statistics-2014 update: a report from the American Heart Association. Circulation. 2014;129(3):e28-e292.

2. Suarez JI, Tarr RW, Selman WR. Aneurysmal subarachnoid hemorrhage. N Engl J Med. 2006;354(4):387-396.

3. Abulhasan YB, Alabdulraheem N, Schiller I, et al. Health care-associated infections after subarachnoid hemorrhage. World Neurosurg. 2018;115:e393-e403.

4. Diringer MN, Bleck TP, Hemphill JC III, et al. Critical care management of patients following aneurysmal subarachnoid hemorrhage: recommendations from the Neurocritical Care Society's Multidisciplinary Consensus Conference. Neurocrit Care. 2011;15(2):211-240.

5. Vergouwen MD, Vermeulen M, van Gijn J, et al. Definition of delayed cerebral ischemia after aneurysmal subarachnoid hemorrhage as an outcome event in clinical trials and observational studies: proposal of a multidisciplinary research group. Stroke. 2010;41(10):2391-2395.

6. de Rooij NK, Rinkel GJ, Dankbaar JW, Frijns CJ. Delayed cerebral ischemia after subarachnoid hemorrhage: a systematic review of clinical, laboratory, and radiological predictors. Stroke. 2013;44(1):43-54.

7. Arakawa Y, Kikuta K, Hojo M, et al. Milrinone for the treatment of cerebral vasospasm after subarachnoid hemorrhage: report of seven cases. Neurosurgery. 2001;48(4):723-730.

8. Duman E, Karakoç F, Pinar HU, et al. Higher dose intraarterial milrinone and intra-arterial combined milrinone-nimodipine infusion as a rescue therapy for refractory cerebral vasospasm. Interv Neuroradiol. 2017;23(6):636-643.

9. Fraticelli AT, Cholley BP, Losser MR, et al. Milrinone for the treatment of cerebral vasospasm after aneurysmal subarachnoid hemorrhage. Stroke. 2008;39(3):893-898.

10. Romero CM, Morales D, Reccius A, et al. Milrinone as a res- cue therapy for symptomatic refractory cerebral vasospasm in aneurysmal subarachnoid hemorrhage. Neurocrit Care. 2009;11(2):165-171.

11. Shankar JJ, dos Santos MP, Deus-Silva L, Lum C. Angiographic evaluation of the effect of intra-arterial milrinone therapy in patients with vasospasm from aneurysmal subarachnoid hemorrhage. Neuroradiology. 2011;53(2):123-128.

12. Lannes M, Zeiler F, Guichon C, Teitelbaum J. The use of milrinone in patients with delayed cerebral ischemia following subarachnoid hemorrhage: a systematic review. Can J Neurol Sci. 2017;44(2):152-160.

13. Lannes M, Teitelbaum J, del Pilar Cortés M, et al. Milrinone and homeostasis to treat cerebral vasospasm associated with subarachnoid hemorrhage: the Montreal Neurological Hospital protocol. Neurocrit Care. 2012;16(3):354-362.

14. Crespy T, Heintzelmann M, Chiron C, et al. Which protocol for milrinone to treat cerebral vasospasm associated with subarachnoid hemorrhage? J Neurosurg Anesthesiol. 2019;31(3):323-329.

15. Abulhasan YB, Alabdulraheem N, Simoneau G, et al. Mortality after spontaneous subarachnoid hemorrhage: causality and validation of a prediction model. World Neurosurg. 2018;112:e799-e811.

16. Yoon DY, Choi CS, Kim KH, Cho BM. Multidetector-row CT angiography of cerebral vasospasm after aneurysmal subarachnoid hemorrhage: comparison of volume-rendered images and digital subtraction angiography. AJNR Am J Neuroradiol. 2006;27(2):370-377.

17. Lao A, Sharma VK, Katz ML, Alexandrov AV. Diagnostic criteria for transcranial doppler ultrasound. In: McGahan JP, Goldberg BB, eds. Diagnostic Ultrasound. 2nd ed. New York: Informa Healthcare; 2008:552-554.

18. Naidech AM, Drescher J, Tamul P, et al. Acute physiological derangement is associated with early radiographic cerebral infarction after subarachnoid haemorrhage. J Neurol Neurosurg Psychiatry. 2006;77(12):1340-1344.

19. Shankar JJ, Tan IY, Krings T, et al. CT angiography for evaluation of cerebral vasospasm following acute subarachnoid haemorrhage. Neuroradiology. 2012;54(3):197-203.

20. Benjamini Y, Hochberg Y. Controlling the false discovery rate: a practical and powerful approach to multiple testing. $J$ $R$ Stat Soc B. 1995;57(1):289-300.

21. Rubin DB. Using propensity scores to help design observational studies: application to the tobacco litigation. Health Serv Outcomes Res Methodol. 2001;2(3-4):169-188.

22. Suwatcharangkoon S, De Marchis GM, Witsch J, et al. Medical treatment failure for symptomatic vasospasm after subarachnoid hemorrhage threatens long-term outcome. Stroke. 2019;50(7):1696-1702.

23. Dorsch NW. Therapeutic approaches to vasospasm in subarachnoid hemorrhage. Curr Opin Crit Care. 2002;8(2):128133.

24. Ehlert A, Schmidt C, Wölfer J, et al. Molsidomine for the prevention of vasospasm-related delayed ischemic neurological deficits and delayed brain infarction and the improvement of clinical outcome after subarachnoid hemorrhage: a single-center clinical observational study. J Neurosurg. 2016;124(1):51-58.

25. Pluta RM. Delayed cerebral vasospasm and nitric oxide: review, new hypothesis, and proposed treatment. Pharmacol Ther. 2005;105(1):23-56.

26. Vergouwen MD, Ilodigwe D, Macdonald RL. Cerebral infarction after subarachnoid hemorrhage contributes to poor outcome by vasospasm-dependent and -independent effects. Stroke. 2011;42(4):924-929.

27. Aldakkan A, Mansouri A, Jaja BN, et al. Predictors of delayed cerebral ischemia in patients with aneurysmal subarachnoid hemorrhage with asymptomatic angiographic vasospasm on admission. World Neurosurg. 2017;97:199-204. 
28. Lazaridis C, Naval N. Risk factors and medical management of vasospasm after subarachnoid hemorrhage. Neurosurg Clin N Am. 2010;21(2):353-364.

29. Lee H, Perry JJ, English SW, et al. Clinical prediction of delayed cerebral ischemia in aneurysmal subarachnoid hemorrhage. J Neurosurg. 2018;130(6):1914-1921.

30. Shapiro S. Milrinone in addition to hyperdynamic therapy in the treatment of vasospasm following aneurysmal subarachnoid hemorrhage. ClinicalTrials.gov. https://clinicaltrials.gov/ ct2/show/NCT02712788. Accessed February 4, 2020.

\section{Disclosures}

The authors report no conflict of interest concerning the materials or methods used in this study or the findings specified in this paper.

\section{Author Contributions}

Conception and design: Abulhasan, Teitelbaum, Angle. Acquisition of data: Abulhasan, Ortiz Jimenez, Teitelbaum. Analysis and interpretation of data: all authors. Drafting the article: Abulhasan, Ortiz Jimenez, Teitelbaum, Simoneau. Critically revising the article: all authors. Reviewed submitted version of manuscript: all authors. Approved the final version of the manuscript on behalf of all authors: Abulhasan. Statistical analysis: Simoneau. Study supervision: Abulhasan.

\section{Supplemental Information Online-Only Content}

Supplemental material is available with the online version of the article.

Supplemental Material. https://thejns.org/doi/suppl/10.3171/ 2020.1.JNS193107.

\section{Correspondence}

Yasser B. Abulhasan: Montreal Neurological Institute and Hospital, Montréal, Quebec, Canada. yasser.abulhasan@hsc.edu.kw 